\title{
$\beta$-Hydroxysteroid Dehydrogenase Type 1 Inhibition Attenuates the Adverse Effects of Glucocorticoids on Dermal Papilla Cells
}

\author{
Sang Eun Lee ${ }^{1}$, Ji Young Choi ${ }^{1}$, Eun Young Lee ${ }^{2}$, Sang Jin Kang ${ }^{2}$, Seung Hun Lee ${ }^{1}$
}

${ }^{1}$ Department of Dermatology and Cutaneous Biology Research Institute, Gangnam Severance Hospital, Yonsei University College of Medicine, Seoul, Korea 2Department of Biotechnology, CHA University, Seongnam, Korea

\section{There are NO conflicts of interest declared in this presentation.}

\section{Introduction}

A role of chronic stress on hair loss

$\checkmark$ Emotional stress is related with several types of hair loss such as telogen effluvium, androgenetic alopecia, female pattern hair loss 2,3

$\checkmark$ Inhibitory effect on hair growth in stress mouse model organ-cultured human scalp hair follicles 4,5

$\checkmark$ Functional hypothalamic-pituitary-adrenal (HPA) axis in normal hair follicles and alopecia

$\checkmark$ Hair follicles have functional peripheral equivalent of HPA axis 5

$\checkmark$ Increased HPA axis tone is observed centrally and peripherally (hair follicles) in mouse model of alopecia6,7

$\checkmark$ Glucocorticoids (GC)

$\checkmark$ Major player in adverse consequence of stress

$\checkmark$ Induces hair follicle regression in in vivo experiment8,9

$\checkmark$ Inhibit proliferation of dermal papilla cells (DPCs) by inducing cell cycle arrest and suppressing the expression of growth factors important in hair follicle growth in DPCs10

$\checkmark 11 \beta$-hydroxysteroid dehydrogenase (11ß-HSD) : a tissue-specific regulator of glucocorticoid response

$\checkmark$ Catalyze the interconversion between hormonally active cortisol and inactive cortisone in cells

$\checkmark 11 \beta$-HSD type 1 (11ß-HSD1)

$\checkmark$ Reductase which activates cortisol from inactive cortisone

$\checkmark$ Keratinocytes, fibroblast, and sebocytes

$\checkmark$ Involved in cell proliferation, wound healing, inflammation, and aging in skin14

$\checkmark$ However, the expression and function of 116-HSD1 in human hair follicles remains unknown.

\section{Objective}

$\checkmark$ To investigate the expression and regulation of 11ß-HSD1 in human DPCs in vitro and in vivo $\checkmark$ To examine whether modulation of 11ß-HSD1 activity can regulate the negative effects of glucocorticoids on DPCs

\section{Material and Methods}

- Immunohistochemistry (IHC) for 11 $\beta$-HSD1

$\checkmark 12$ Paraffin-embedded normal human scalp skin samples

$\checkmark$ Primary anti-11ß-HSD1 antibody (rabbit, polyclonal, 1:100 dilution, Abcam, Cambridge, UK)

$\checkmark$ Secondary horseradish peroxidase-conjugated antibody

- Cell culture and reagents

$\checkmark$ DPCs of human origin [PromoCell GmbH (Heidelberg, Germany)]

$\checkmark$ Cortisol [Sigma Aldrich (H6909, St Louis, MI, U.S.A.)] and a selective inhibitor of 11ß-HSD1, 385581 [Merck (Darmstadt, Germany)] dissolved in DMSO, a final concentration of $0.1 \mu \mathrm{M}$

- Immunofluorescence Analysis of Ki-67 Expression

- Western blot analysis for 11ß-HSD1, Wnt5a, alkaline phosphatase (ALP), and vascular endothelial growth factor (VEGF)

- Statistic analysis

$\checkmark$ Two-tailed Student $t$ test or one-way ANOVA with Dunnett's post test Graph Pad In Stat version 3.00 software (Graph Pad Software, San Diego, CA, USA)

\section{Results}

-Human scalp hair follicles express 11ß-HSD1 in ORS, matrix keratinocytes, and dermal papilla

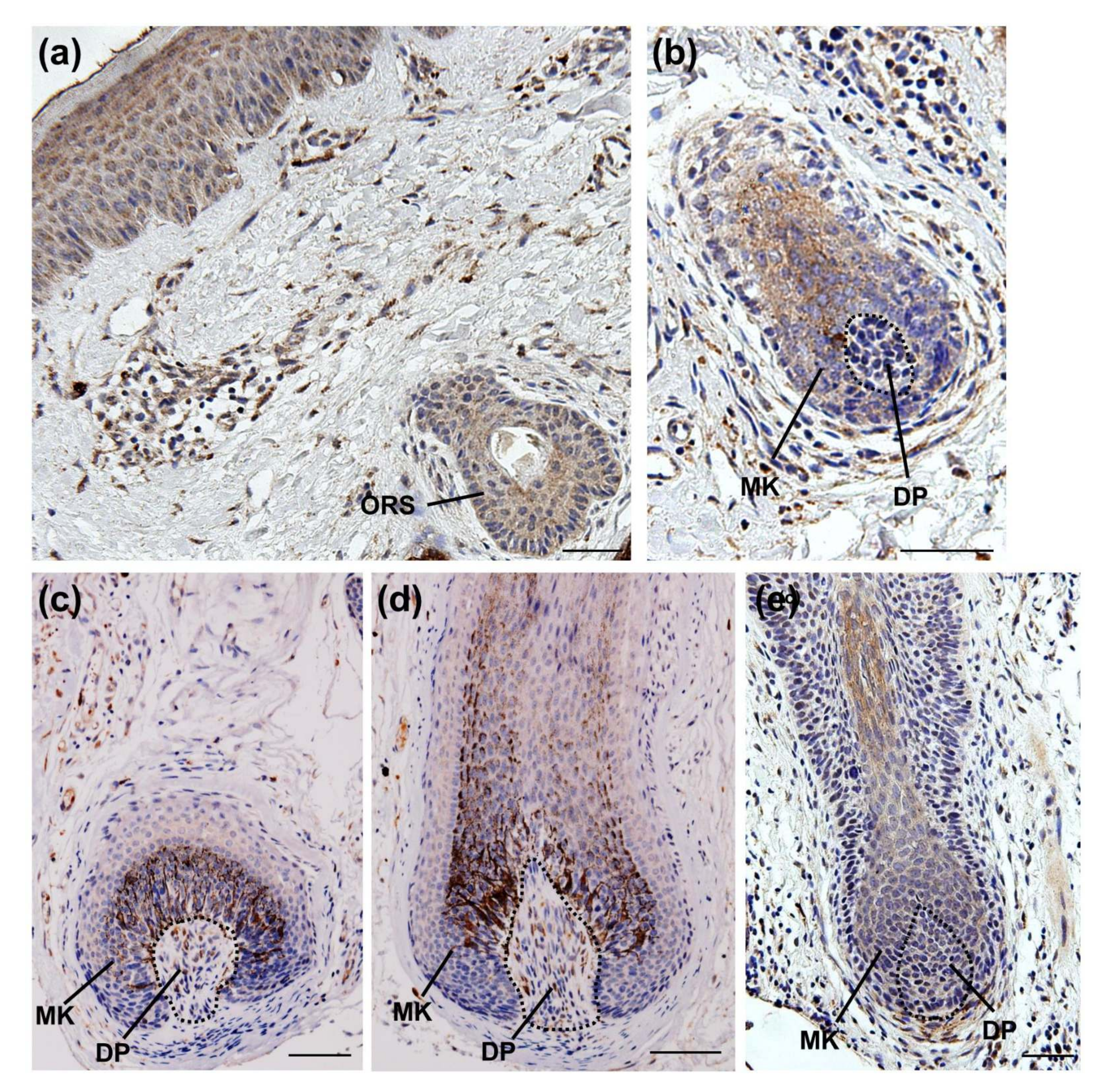

Figure 1. Expression of 11ß-HSD1 by immunohistochemical staining in human scalp hair follicles. 11ß-HSD1 immunoreactivity was found in ORS keratinocytes (a), the matrix keratinocytes (MK) (b and c; arrow, original magnification $x$ $200)$, and in the DP in situ ( $b$ and c, original magnification $\times 200)$.

- Human DPCs express 11ß-HSD1 protein in vitro and GC upregulates 11ß-HSD1 protein expression in cultured human dermal papilla cells (hDPCs)
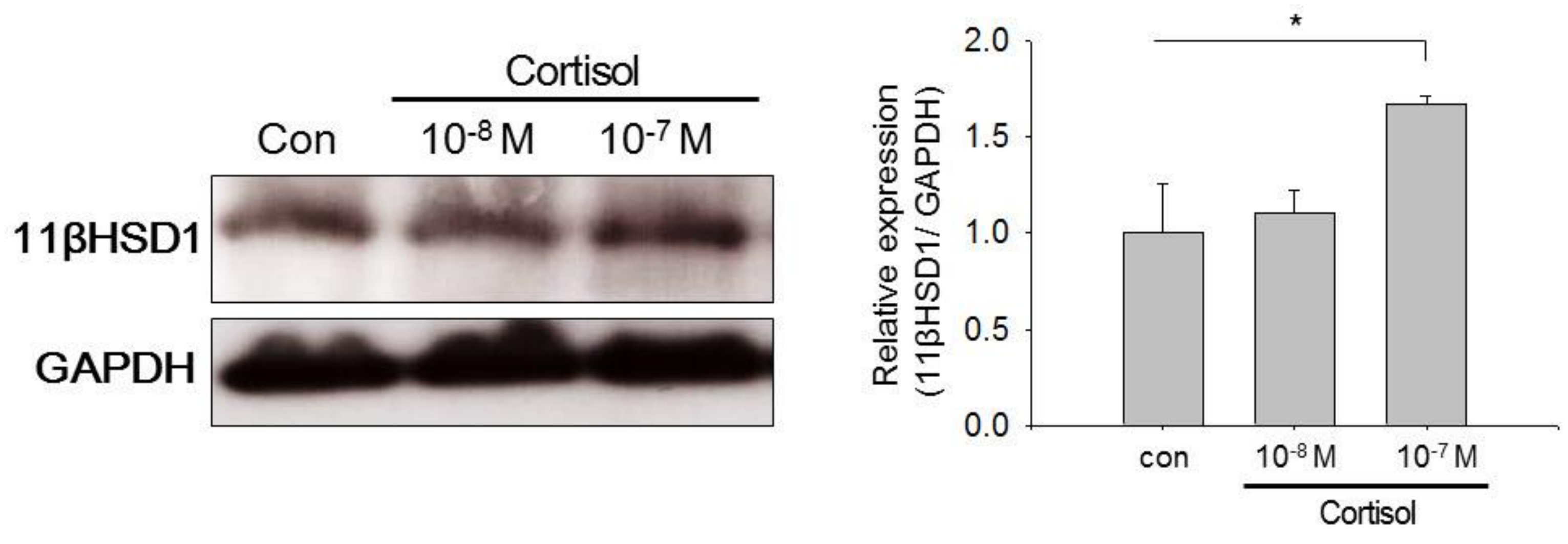

Figure 2. Western blot analysis of 11ß-HSD1 expression in unstimulated and cortisol-stimulated human DPCs. Bars show the result of densitometric analysis of the 11ß-HSD1 protein band relative to the corresponding GAPDH protein band. Results are presented as the mean $\pm S D$. $\left({ }^{*} p<0.05\right)$.

- Inhibition of $11 \beta-H S D 1$ by selective inhibitor partially reverses the GC-induced decrease in the proliferation of hDPCs
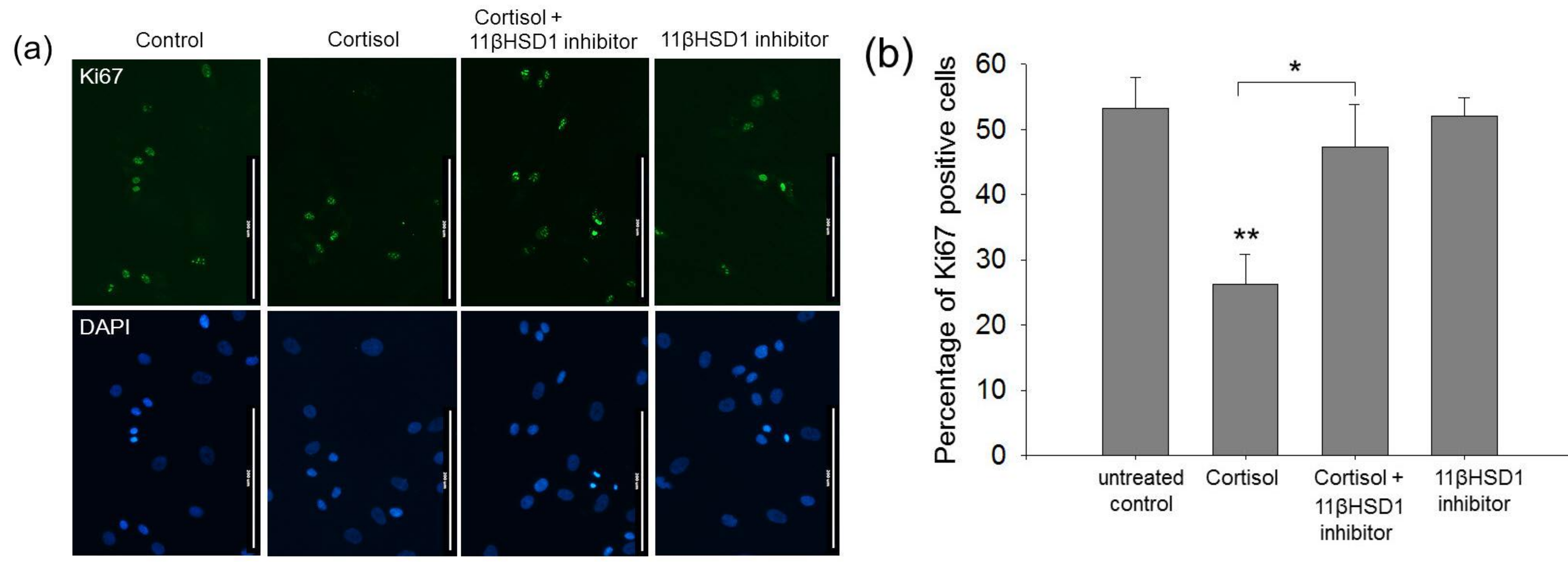

Figure 3. The effect of the selective 11ß-HSD1 inhibitor on the proliferation in cortisol-stimulated hDPCs. (a) hDPCs were treated with 10-7 M cortisol for 48 hours with/without $30 \mathrm{~min}$ pretreatment with 385581 (100 nmol L-1), and immunofluorescent staining was performed with anti-Ki-67 antibody. (b) Percentage of Ki-67-positive human DPCs (green in nuclei) under at least 5 high power fields was counted. $\left({ }^{*} p<0.05,{ }^{* *} p<0.01\right)$.

- The selective $11 \beta-H S D 1$ inhibitor partially reverses the glucocorticoid-induced decrease in the expression of dermal papilla markers and growth factor in DPCs

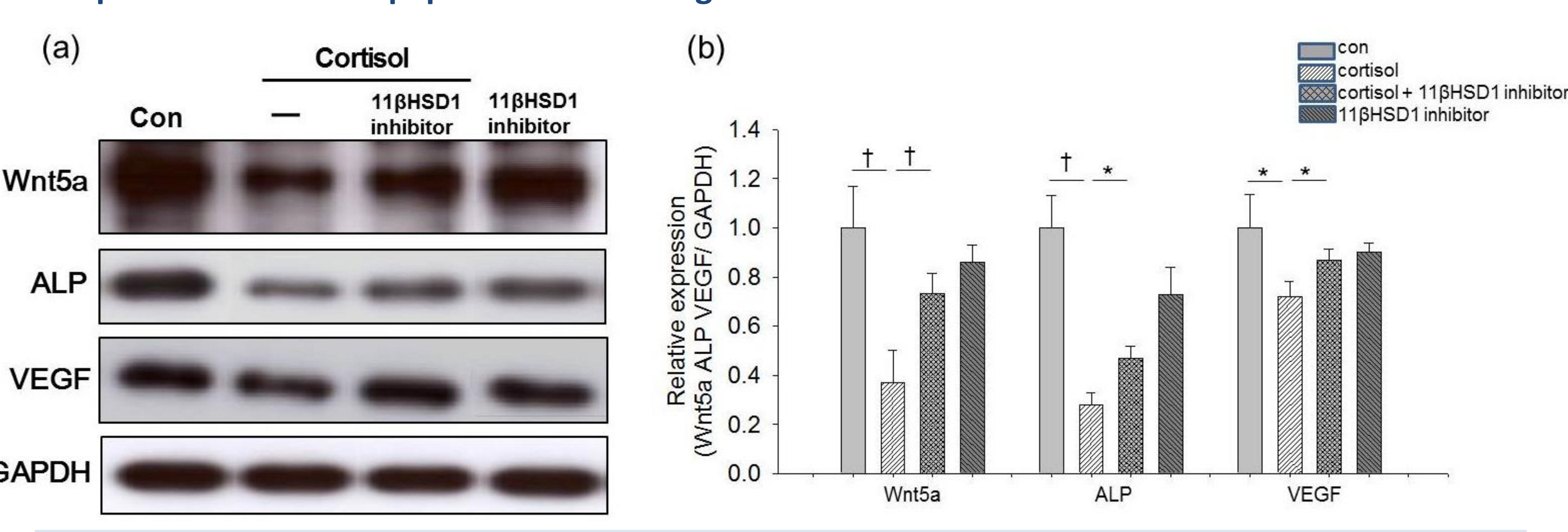

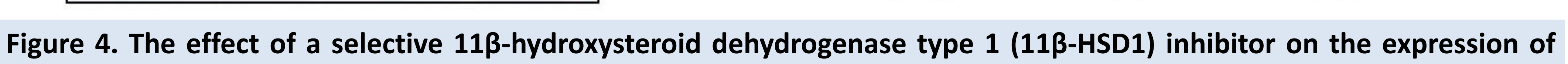
Wnt5a, ALP, and VEGF in cortisol-stimulated human dermal papilla cells. Human DPCs were pre-treated with $100 \mathrm{nmol} L-1$ 11ß-HSD1 inhibitor for 30 minutes and stimulated with 10-7 M cortisol for 48 hours. (a) After stimulation, the expression of Wnt5a, ALP, and VEGF was analyzed by Western blot. (b) Bars show the results of densitometric analysis relative to the corresponding GAPDH protein band. Results are presented as mean \pm SD. $\left({ }^{*} p<0.05,{ }^{* *} p<0.01\right)$.

\section{Summary \& Conclusions}

$\checkmark$ This is the first demonstration of 11ß-HSD1 expression in human DPCs both in situ and in vitro at protein level and the regulation of 11ß-HSD1 by cortisol in human DPCs.

$\checkmark$ Cortisol suppresses the expression of dermal papilla biomarkers involved in the maintenance of human dermal papilla properties, including Wnt5a and ALP, a ubiquitous dermal papilla marker.

$\checkmark$ Inhibition of 11ß-HSD1 can prevent glucocorticoid-induced suppression of proliferation, growth factor expression, and the expression of dermal papilla markers in human DPCs.

We can suggest that DPCs are not only the target cells for GCs but also metabolize and synthesize active form of GC via the presence of 116-HSD1.

Our data provide insights into the mechanisms underlying chronic stress-related hair loss and suggest that 116-HSD1 inhibitors can potentially be used to prevent stress-related hair loss

\section{References}

$\checkmark$ Peters EM, Arck PC, Paus R. Hair growth inhibition by psychoemotional stress: a mouse model for neural mechanisms in hair growth control. Exp Dermatol 2006;15:1-13.

$\checkmark$ Zhang X, Yu M, Yu W, Weinberg J, Shapiro J, McElwee KJ. Development of alopecia areata is associated with higher central and peripheral hypothalamic-pituitary-adrenal tone in the skin graft induced C3H/HeJ mouse model. J Invest Dermatol 2009;129:1527-38.

$\checkmark$ Tomlinson JW, Walker EA, Bujalska IJ, Draper N, Lavery GG, Cooper MS, et al. 11betahydroxysteroid dehydrogenase type 1: a tissue-specific regulator of glucocorticoid response. Endocr Rev 2004; 25:831-66. 